\title{
Numerical Studies Of the Upstream Flow Field Around A Horizontal Axis Wind Turbine
}

\author{
Hamidreza Abedi* \\ Chalmers University of Technology, 412 96, Gothenburg, Sweden \\ Lars Davidson, ${ }^{\dagger}$ \\ Chalmers University of Technology, 412 96, Gothenburg, Sweden \\ Spyros Voutsinas, ${ }^{\ddagger}$ \\ National Technical University of Athens, Athens, Greece
}

\begin{abstract}
The aerodynamics of a wind turbine is governed by the flow around the rotor. Prediction of the velocity field, both upstream and downstream, is one of the challenges for wind turbine performance in terms of the aerodynamic loads and the generated power at different operational conditions. For simplicity, the wind velocity at the rotor plane is assumed to be equal to far upstream flow where the interaction of the rotor blades with upstream flow, close to the rotor plane, is not taken into account. This paper aims to study the effect of the rotor blade azimuthal position and the trailing wake, on upstream and downstream flow near to the rotor plane. For this purpose, an in-house Vortex Lattice Free Wake (VLFW) code, based on the potential, inviscid and irrotational flow, is developed. The results are compared with the MEXICO wind tunnel measurements. They show that the wind speed decreases in the axial direction upstream the rotor plane because of the induced velocity field by the rotor blades and the trailing wake vortices. This leads to a power reduction of the wind turbine. Furthermore, contrary to the traditional actuator disk model, the VLFW simulations predicts a tangential velocity component upstream the rotor due to the blade rotation which is in agreement with the measurement data. Finally, it is found that the flow field downstream and upstream the rotor blades depends on the blade azimuthal direction.
\end{abstract}

\section{Nomenclature}

BEM Blade Element Momentum

CFD Computational Fluid Dynamics

$\Gamma \quad$ Circulation, $\mathrm{m}^{2} / \mathrm{s}$

$\vec{V} \quad$ Velocity vector, $\mathrm{m} / \mathrm{s}$

$\Omega$ Rotational velocity, $\mathrm{rad} / \mathrm{s}$

$\vec{r} \quad$ Position vector, $\mathrm{m}$

$\gamma \quad$ Vorticity distribution per length, $\mathrm{m} / \mathrm{s}$

$t$ time, s

$\vec{V}_{\infty}$ free stream velocity vector, $\mathrm{m} / \mathrm{s}$

$u \quad$ Velocity component in the $x$ direction, $\mathrm{m} / \mathrm{s}$

$v \quad$ Velocity component in the $y$ direction, $\mathrm{m} / \mathrm{s}$

$w \quad$ Velocity component in the $z$ direction, $\mathrm{m} / \mathrm{s}$

Subscript

$i \quad$ Panel index

$j \quad$ Panel index

T.E. Trailing edge

ind Induced

${ }^{*}$ Ph.D. Candidate, Division of Fluid Dynamics, Department of Applied Mechanics

${ }^{\dagger}$ Professor, Division of Fluid Dynamics, Department of Applied Mechanics

${ }^{\ddagger}$ Associate Professor, Fluid Section, School of Mechanical Engineering 


$\begin{array}{ll}\text { rot } & \text { Rotational } \\ W & \text { Wake } \\ \text { tot } & \text { Total }\end{array}$

\section{Introduction}

Among renewable clean energy sources, wind is regarded as the least destructive to the environment. The exponential growth of wind turbines all around the world, its general acceptance among people, the demand and its worthwhileness makes it apt for research, especially to enhance its performance. According to data provided by the Renewables Global Status Report in 2013, wind capacity increased globally by 19\%, the increase being 45 GW, and it reached to $283 \mathrm{GW}$.

There are different methods for modelling the aerodynamics of a wind turbine depending on levels of complexity and accuracy, such as the Blade Element Momentum (BEM) theory and solving the Navier-Stokes equations using Computational fluid Dynamics (CFD). Today, the BEM method is used extensively to analyze the aerodynamic performance of a wind turbine. The BEM model is based on a steady and homogeneous flow assumption and that aerodynamic loads act on an actuator disc instead of a finite number of blades. The BEM method is computationally fast and is easily implemented, but it is acceptable only for a certain range of flow conditions. ${ }^{1}$ A number of empirical and semi-empirical correction factors have been added to the BEM in order to increase its application range, such as yaw misalignment, dynamic inflow, finite number of blades and blade cone angle ${ }^{2}$ but they are not relevant to all operating conditions and are often incorrect at high tip speed ratios where wake distortion is significant. ${ }^{3}$

In the BEM theory, the rotor blades are modeled as a 1D ideal rotor disc which acts as a drag device (there is no rotational velocity component in the wake). According to the $1 \mathrm{D}$ ideal rotor model, the rotor disc makes the wind speed to gradually decrease from $V_{\infty}$ far upstream of the rotor to $V_{\text {rotor }}$ at the rotor plane and to $V_{\text {wake }}$ in the wake. ${ }^{1}$ The fractional wind velocity reduction between far upstream and the rotor plane is called the axial induction factor. In other words, the axial velocity deficit due to the rotor blades is referred to as the axially induced velocity at the rotor, ${ }^{4}$ and it is expressed by the axial induction factor. Therefore, the 1D ideal rotor model assumes that the velocity field around the rotor blades consists of only free stream and the induced velocities in which half of the flow reduction due to the axially induced velocity occurs at the rotor plane, and the rest occurs far downstream the rotor. However, in calculations based on the BEM method, it is assumed that the wind velocity close to the rotor plane is equal to the far upstream. Therefore, the gradual reduction of wind velocity between the far upstream and close to the rotor plane is not taken into account.

The 1D ideal disk theory was extended for the rotating disk on the basis of the ideal horizontal axis wind turbine with wake rotation. According to the ideal horizontal axis wind turbine with wake rotation, the angular momentum which is added to the air flow due to the blade rotation, makes the flow downstream of the rotor to rotate contrary to the rotating direction of the blades. As a consequence, the rotational velocity of the air increases when it passes over the rotor plane compared with the rotor angular velocity. This small rise in the air angular velocity at the rotor plane is called tangential induction factor. ${ }^{4}$

Combination of the 1D ideal disk theory and the ideal horizontal axis wind turbine with wake rotation leads to the fact that the induced velocity across the rotor includes both the axial and the tangential components. However, it cannot give any information about the flow field upstream the rotor, especially close to the rotor plane. Furthermore, the effect of the rotor blade position in the azimuthal direction and the trailing wake on the flow field upstream and downstream of the rotor blade is not taken into account.

Apart from the BEM and the CFD methods, the vortex method which is based on the potential, inviscid and irrotational flow can be used to predict the aerodynamic performance of wind turbines. It has been widely used for aerodynamic analysis of airfoils and aircrafts. Compared with the BEM method, the vortex method is able to provide more physical solutions for attached flow conditions with boundary layer corrections, and it is also valid over a wider range of turbine operating conditions. Although it is computationally more expensive than the BEM method, it is still feasible as an engineering method.

In the vortex methods, the trailing and shed vortices are modeled by either vortex particles or vortex filaments moving either freely, known as free wake, ${ }^{5-9}$ or restrictedly by imposing the wake geometry known as prescribed wake. $^{10,11}$ The free wake model, which is the most computationally expensive vortex method, is able to predict the wake geometry and loads more accurately than the prescribed wake. In addition, it can be applied for both axisymmetric and asymmetric upstream flow conditions. However, its application is limited to attached flow and it must be linked to tabulated airfoil data to predict air loads in the presence of drag and flow separation. 
In addition to the axially and tangentially induced velocities, there is a radially induced velocity component which cannot be estimated on the basis of the ideal horizontal axis wind turbine with wake rotation and the 1D ideal rotor disc theories. Moreover, both of these theories only estimate the axially and the tangentially induced velocities at the rotor plane, and they cannot predict the induced velocity field upstream the rotor plane, particularlly close to the rotor plane.

The time-marching vortex lattice free wake is used to study the effect of the rotating blades and the trailing wake vortices on the upstream and the downstream flow field around a horizontal axis wind turbine. The results are compared with the Mexico project experimental data. ${ }^{12}$ The MEXICO wind turbine measurements were carried out in 2006 in the Large Scale Low Speed Facility (LLF) of the German Dutch Wind Tunnels (DNW).

\section{Model}

\section{II.A. Assumptions}

In this study, the upstream flow is set to be uniform, both in time and space, and is perpendicular to the rotor plane (parallel to the rotating axis). However, it can be either uniform or non-uniform (varying both in time and space). The blades are assumed to be rigid. Therefore, the elastic effects of the blades are neglected. Moreover, because of

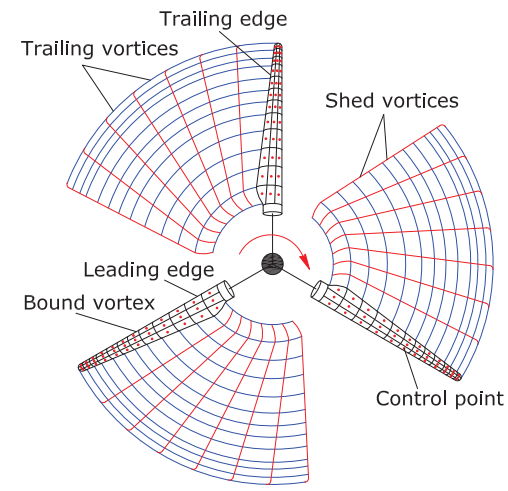

Figure 1. Schematic of vortex lattice free wake

the large circulation gradients $(d \Gamma / d r)$ near the tip and the root of the rotor blade, the cosine rule for the blade radial segmentation $^{2}$ is used where the blade elements are distributed at equi-angle increments in the spanwise direction.

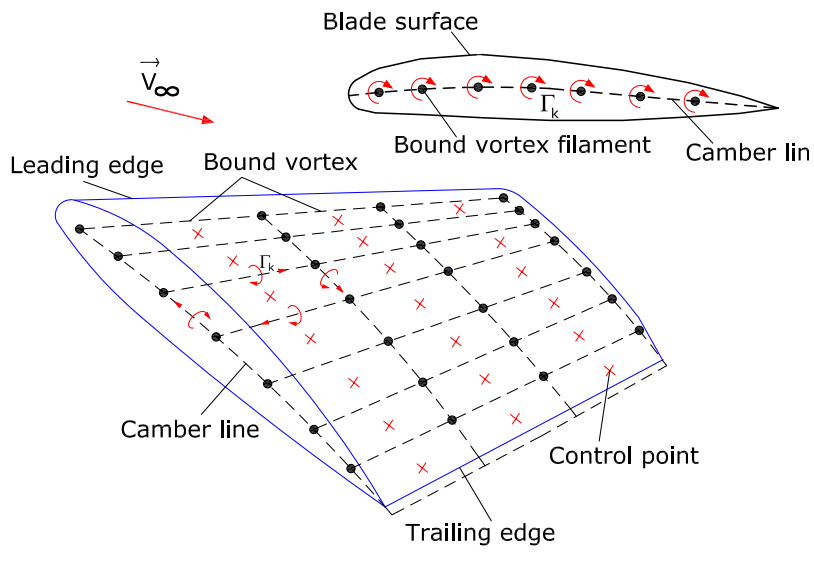

Figure 2. Lifting surface and vortex panels construction

\section{II.B. Vortex Lattice Free Wake (VLFW)}

The vortex lattice method is based on the thin lifting surface theory of vortex ring elements, ${ }^{13}$ in which the blade surface is replaced by vortex panels that are constructed based on the airfoil camber line of each blade section (see figure 2). To take the blade surface curvature into account, the lifting surface is divided into a number of panels, both 
in the chordwise and spanwise directions, where each panel contains the vortex ring with strength $\Gamma_{i j}$ in which $i$ and $j$ indicate panel indices in the chordwise and spanwise directions, respectively. The strength of each blade bound vortex ring element, $\Gamma_{i, j}$, is assumed to be constant, and the positive circulation is defined on the basis of the right-hand rotation rule.

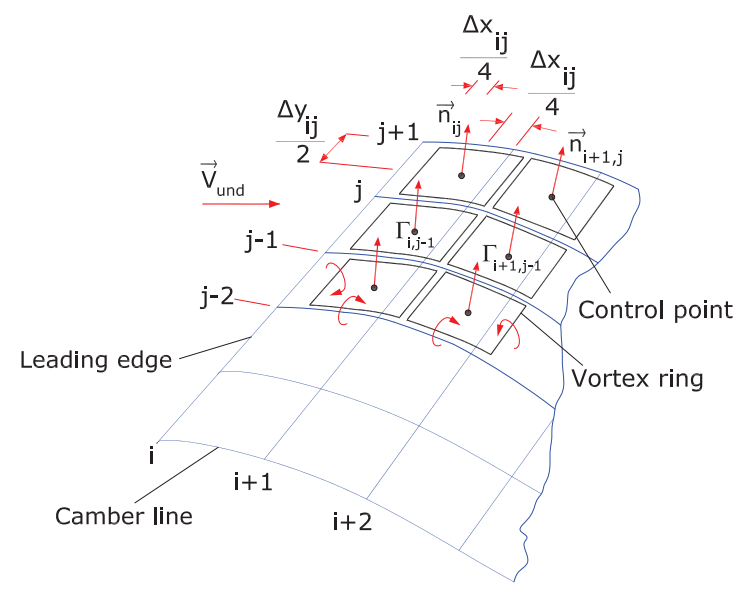

Figure 3. Numbering procedure

In the vortex lattice free wake model, a finite number of vortex wake elements move freely based on the local velocity field, and contrary to the prescribed wake model, allowing wake expansion as well. Each vortex filament contains two points; one at the head (A) and another at the tail (B). They are known as Lagrangian markers, where the induced velocity components are calculated using the Biot-Savart law; their movements give rise to the wake deformation. The vortex flow theory assumes that the trailing and shed wake vortices extend to infinity. However, since the effect of the induced velocity field by the far wake is small on the rotor blade, the wake in the present study extends only four diameters downstream of the wind turbine rotor plane.

In order to fulfill the 2D Kutta condition (which can be expressed as $\gamma_{T . E .}=0$ in terms of the strength of the vortex sheet where the T.E. subscripts denotes the trailing edge) the leading segment of a vortex ring is located at the $1 / 4$ panel length (see figure 3). The control point of each panel is located at $3 / 4$ of the panel length meaning that the control point is placed at the center of the panel's vortex ring.

The wake elements which induce a velocity field around the blade are modeled as vortex ring elements, and they are trailed and shed based on a time-marching method. To satisfy the 3D trailing edge condition for each spanwise section, the strength of the trailing vortex wake rings must be equal to the last vortex ring row in the chordwise direction $\left(\Gamma_{T . E .}=\Gamma_{\text {Wake }}\right)$.

The flow tangency condition at each blade's control point must be specified to find the blade bound vortices strength $\left(\Gamma_{i, j}\right)$ at each time step. The velocity components at each blade's control point include the free stream $\left(\mathbf{V}_{\infty}\right)$, rotational $(\Omega \mathbf{r})$, blade vortex rings self-induced $\left(\mathbf{V}_{\text {ind,bound }}\right)$ and wake induced $\left(\mathbf{V}_{\text {ind,wake }}\right)$ velocities where $\mathbf{V}_{\infty}, \Omega \mathbf{r}$ and $\mathbf{V}_{\text {ind,wake }}$ are known at each time step.

The blade is assumed to be rigid, hence the blade self-induced components, called influence coefficients, are constant at each time step, and they are computed only once. However, if the blade would be modeled as a flexible blade, they would need to be calculated at each time step.

At the first time step (see figure 4), there are no free wake elements. At the second time step (see figure 5), when the blade is rotating, the first wake panels are shed. Their strength is equal to the bound vortex circulation of the last row of the blade vortex ring elements (Kutta condition) located at the trailing edge at the previous time step, which means that $\Gamma_{W_{t_{2}}}=\Gamma_{T . E ., t_{1}}$, where the $W$ and T.E. subscripts represent the wake and the trailing edge, respectively. At the second time step, the strength of the blade bound vortex rings is calculated by specifying the flow tangency boundary condition where, in addition to the blade vortex ring elements, the contribution of the first row of the wake panels is considered. The vortex wake elements are trailed and shed at each time step, where their strengths remain constant (Kelvin theorem), and their corner points are moved based on the governing equation (Eq.(1)) by the local velocity field, including the wind velocity and the induced velocity by all blade and wake vortex rings (see figures 4 and 5). The governing equation for the wake geometry is

$$
\frac{d \vec{r}}{d t}=\vec{V}(\vec{r}, t) \quad \vec{r}(t=0)=\overrightarrow{r_{0}}
$$




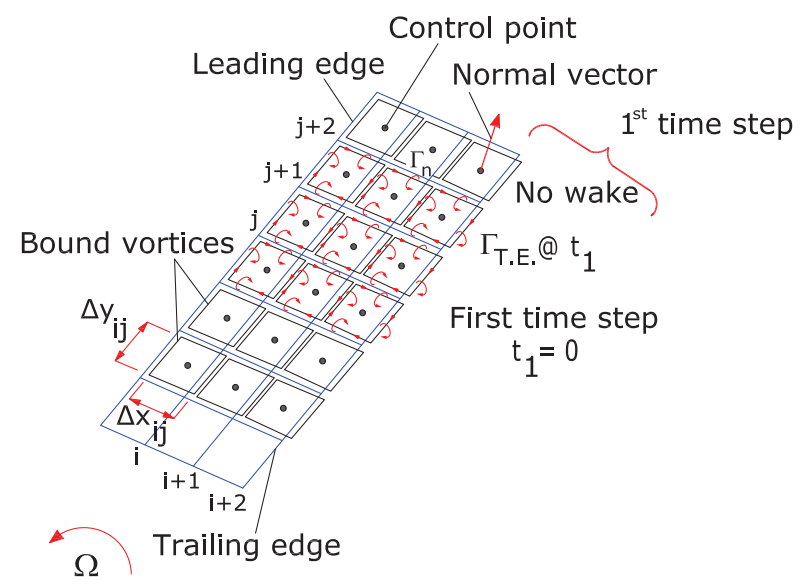

Figure 4. Schematic of wake evolution at the first time step

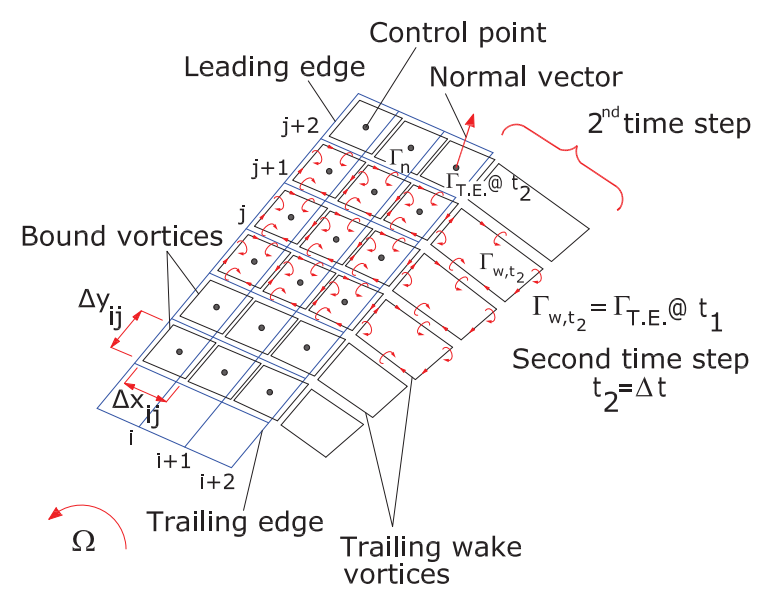

Figure 5. Schematic of wake evolution at the second time step 
where $\vec{r}, \vec{V}$ and $t$ denote the position vector of a Lagrangian marker, the total velocity field and time. The total velocity field, expressed in the rotating reference frame i.e. $\vec{V}_{r o t}=0$, can be written as

$$
\vec{V}_{\text {tot }}=\vec{V}_{\infty}+\vec{V}_{\text {ind,blade }}+\vec{V}_{\text {ind,wake }}
$$

Different numerical schemes may be used for Eq.(1) such as the explicit Euler method, the implicit method, Adams-Bashforth method and the Predictor-Corrector method. The numerical integration scheme must be considered in terms of the accuracy, stability and computational efficiency. Here, the first-order Euler explicit method is used as

$$
\vec{r}_{t+1}=\vec{r}_{t}+\vec{V}_{t o t}\left(\vec{r}_{t}\right) \Delta t
$$

where $\vec{V}$ is taken at the old time step.

\section{Results}

The 3-bladed MEXICO wind turbine ${ }^{12}$ is used in the simulation. The operating conditions at which the measurement has been done ${ }^{12}$ are: $V_{\text {tunnel }}=10.01 \mathrm{~m} / \mathrm{s}, \Omega=44.45 \mathrm{rad} / \mathrm{s}, \rho=1.245 \mathrm{~kg} / \mathrm{m}^{3}$ where $V, \Omega$ and $\rho$ denote the free stream velocity, rotational velocity and the air density, respectively. Moreover, the rotor diameter, the blade length and the blade pitch angle are $4.5 \mathrm{~m}, 2.04 \mathrm{~m}$ and -2.3 degrees, respectively. ${ }^{12}$ In the vortex method simulations made with

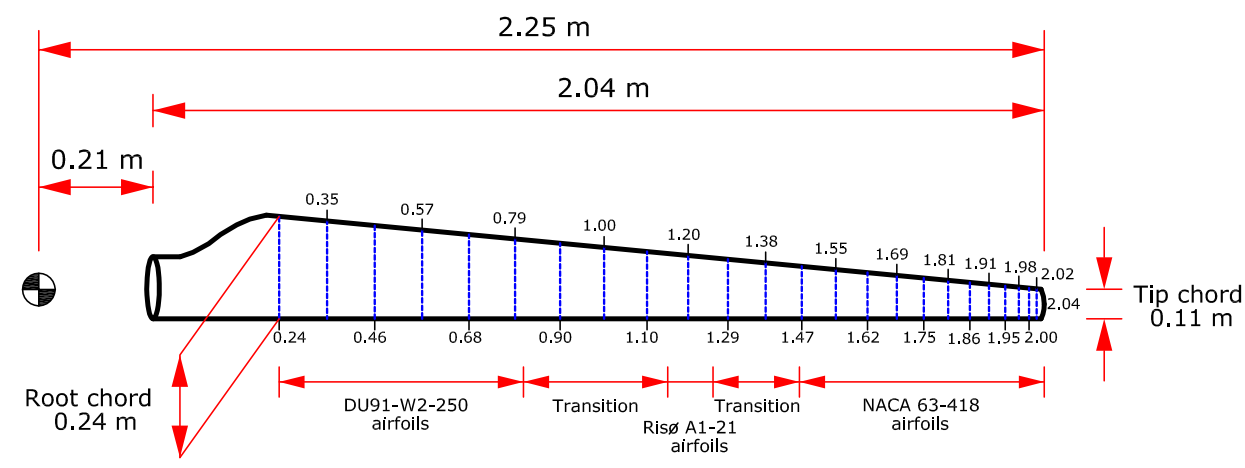

Figure 6. Radial distribution of blade elements

VLFW, the blade is discretized with 24 spanwise sections with fine tip resolution (see figure 6) and 10 equally spaced chordwise sections. 10 degrees in the azimuthal direction is employed for the wake segmentation. ${ }^{14}$ The wake length is truncated after 4 rotor diameters which is based on a grid-sensitivity study according to Ref. 14. It is assumed that the wake vortex filament core radius is constant and is equal to $0.1 \mathrm{~m}$. The free stream is assumed to be uniform, steady and perpendicular to the rotor plane similar to the measurement condition. Figures (7) and (8) show the schematic of PIV measurements on the MEXICO turbine wind turbine done for radial traverse in the horizontal plane at different clock positions.

For the radial traverses, the velocity field is measured both upstream and downstream of the rotor at different blade positions (see figure 7). The horizontal plane at the 9 o'clock position extends from $z=-0.30 \mathrm{~m}$, upstream of the rotor, to $z=+0.30 \mathrm{~m}$, downstream of the rotor where $x=0$ is in the rotor plane. In addition, the horizontal plane at the 9 o'clock position (see figure 8) extends from $r=1.15 \mathrm{~m}$ to $r=2.75 \mathrm{~m}$ (line $\mathbf{A B}$ and $\mathbf{C D}$ ) where $r=0$ is in the rotor centre. $^{12}$ Different velocity components are measured along AB and $\mathbf{C D}$. There are two azimuthal angles, 20 and 40 degrees, where the rotor blade passes through the measurement plane (see figure 7 ).

As described in the first paragraph of this section, the comparison of the velocity field, between the simulation and the experimental data, has been done for the upstream axial flow equal to $10 \mathrm{~m} / \mathrm{s}$. However, the presented experimental data show that the upstream flow which hits and passes through the rotor blades is less than $10 \mathrm{~m} / \mathrm{s}$ (around $9 \mathrm{~m} / \mathrm{s}$ ). According to Ref. 15, one of the reason for this deficiency may be because of the streamwise velocity which is reduced in open type wind tunnel employed in the experimental investigation.

According to figures 9, 10 and 11, there is a good agreement between the simulation and experimental data. As can be seen, the simulation overestimates the axial velocity compared with measurements for both upstream and downstream of the PIV sheet. However, there is a constant difference between the calculated axial velocity profile by simulation and the measurement data which may be due to the open wind tunnel as mentioned in the previous paragraph. 

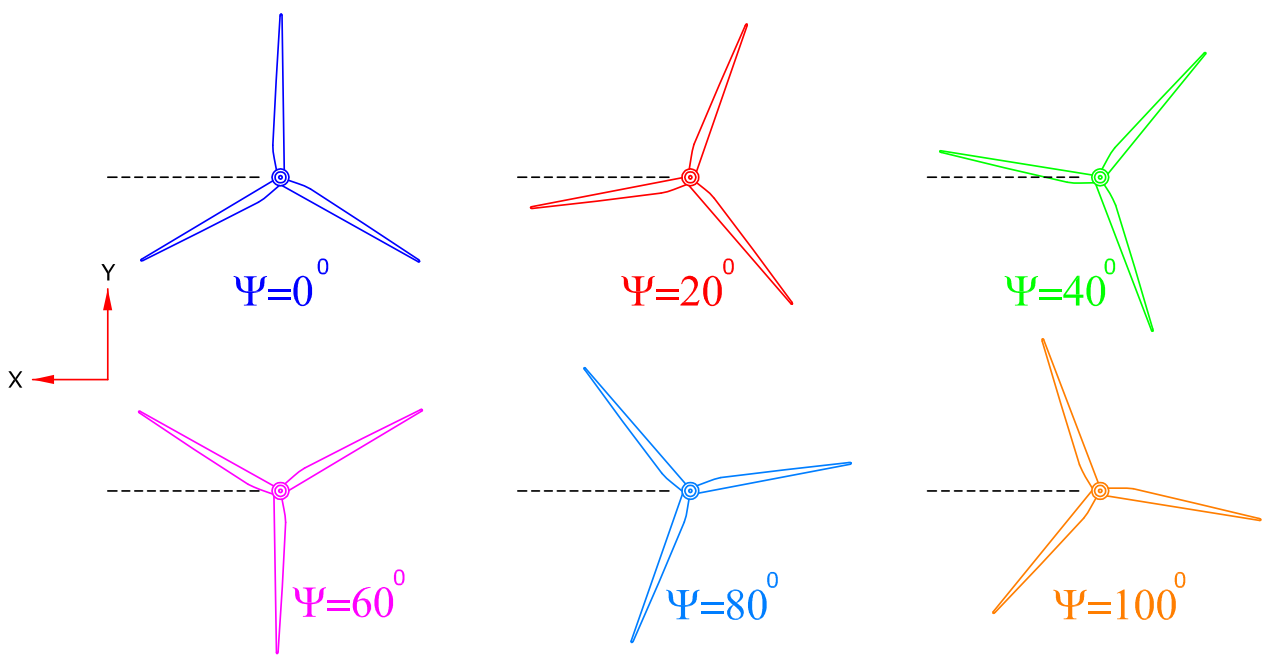

Figure 7. Position of blade 1 at different angles

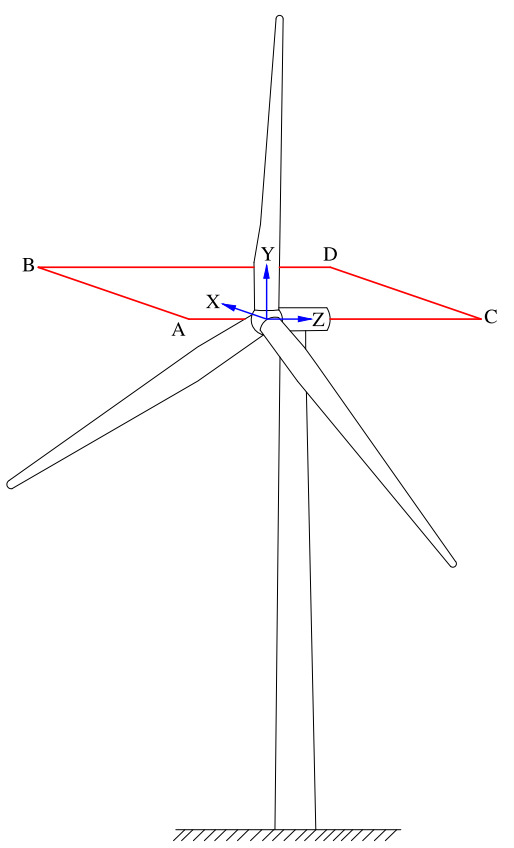

Figure 8. PIV measurement sheet at radial traverses 

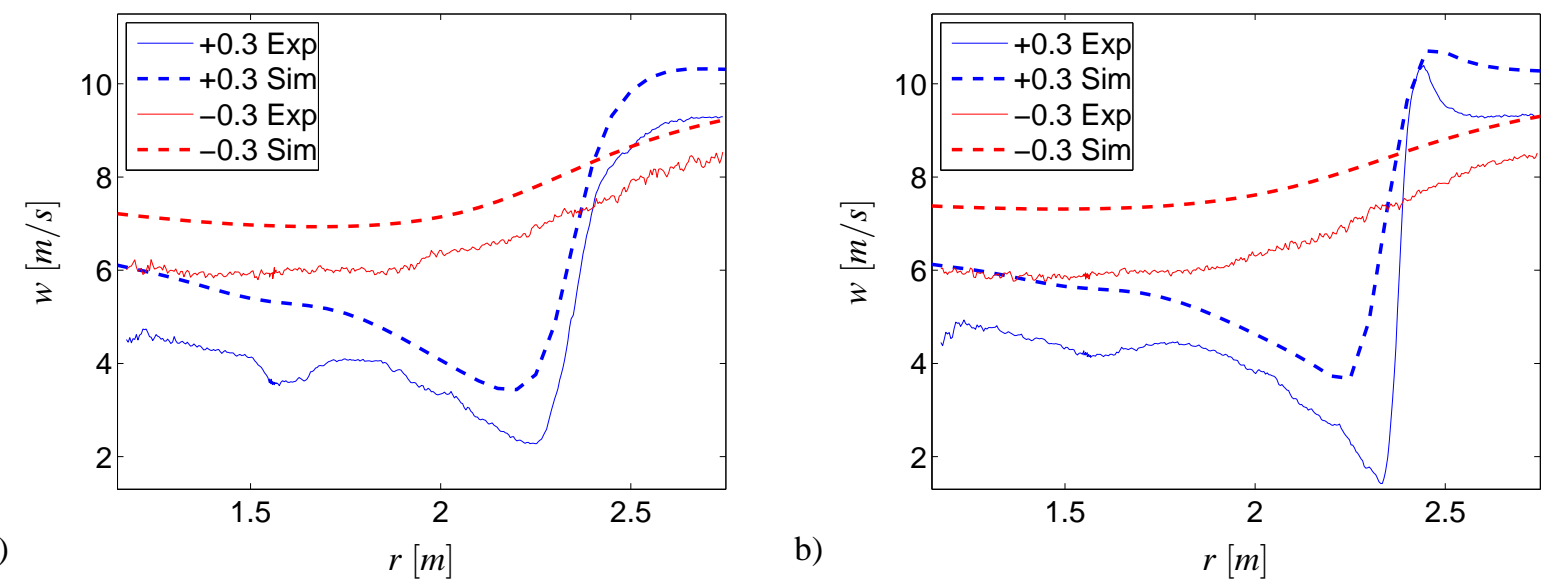

Figure 9. Axial velocity (z direction) upstream (-0.3m) and downstream (+0.3m) of the rotor, blade position at $0^{\circ}$ and $20^{\circ}$, Exp: Experiment, Sim: Simulation

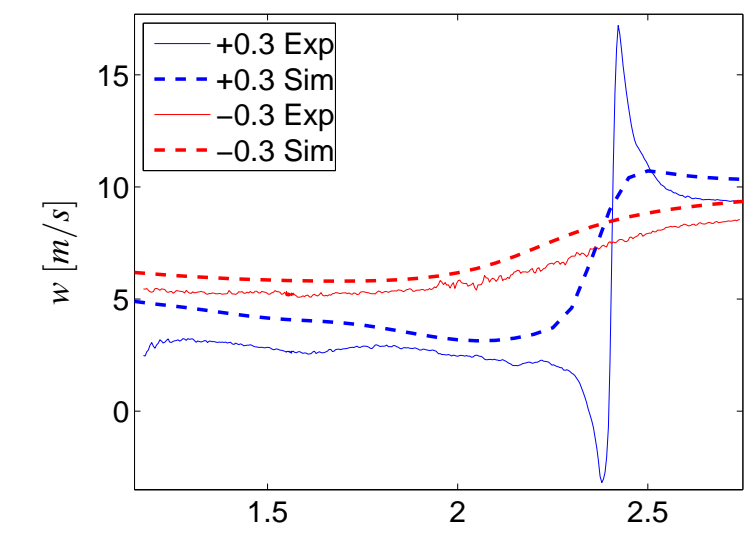

a) $r[m]$

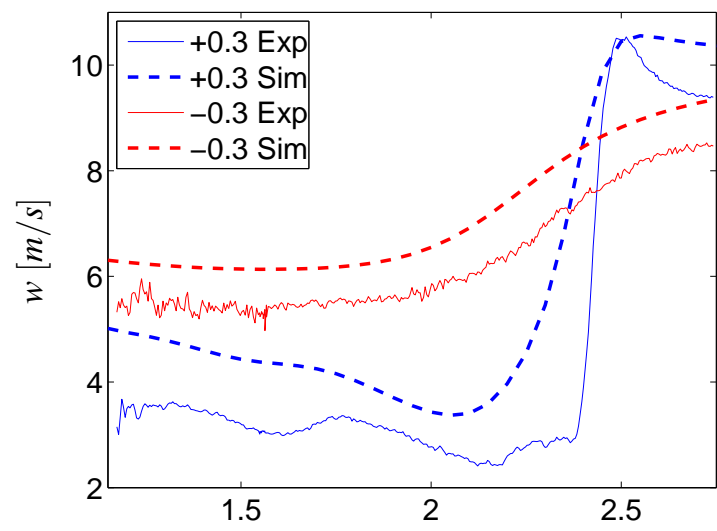

b)

Figure 10. Axial velocity (z direction) upstream (-0.3m) and downstream (+0.3m) of the rotor, blade position at $40^{\circ}$ and $60^{\circ}$, Exp: Experiment, Sim: Simulation

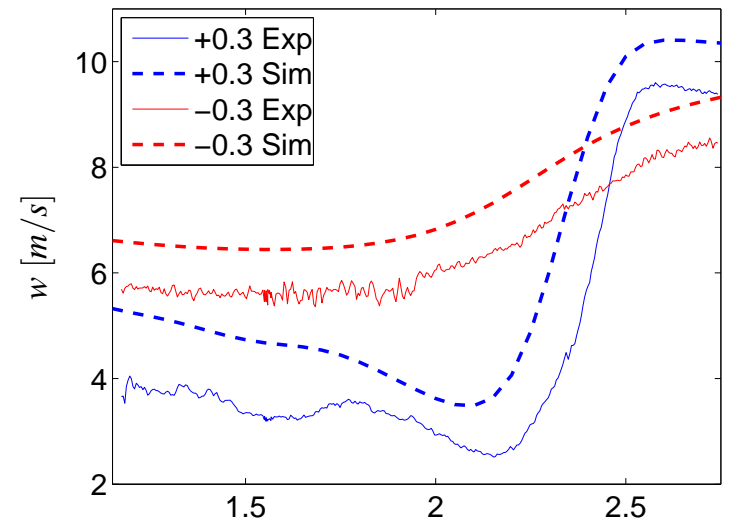

a)

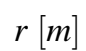

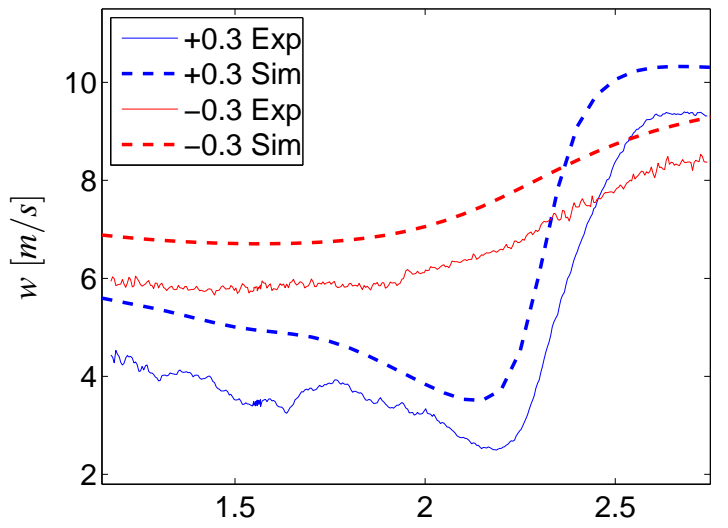

b)

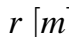

Figure 11. Axial velocity (z direction) upstream (-0.3m) and downstream (+0.3m) of the rotor, blade position at $80^{\circ}$ and $100^{\circ}$, Exp: Experiment, Sim: Simulation 
Figures 9, 10 and 11 display how the presence of the tip vortex makes a significant change in the axial velocity component $(w)$ in the blade tip region. Moreover, downstream of the rotor blade, close to the blade tip region, both simulation and measurement data show the sudden change in the axial velocity. The minimum peak is associated with tip vortex position which influences significantly the flow behind the rotor. In addition, figures 9,10 and 11 show that the tip vortex position varies with respect to the azimuthal angle showing the complex mechanism of the merging vortex sheet close to the tip. One of the aerodynamic features of a horizontal axis wind turbine is the wake expansion which is directly related to the radius of tip vortex position downstream of the rotor blades. The radial position of minimum axial velocity for the measurement data occurs in a slightly larger radial position than in the simulation. This means that the simulation predicts a smaller wake expansion compared with the experiments. ${ }^{15}$

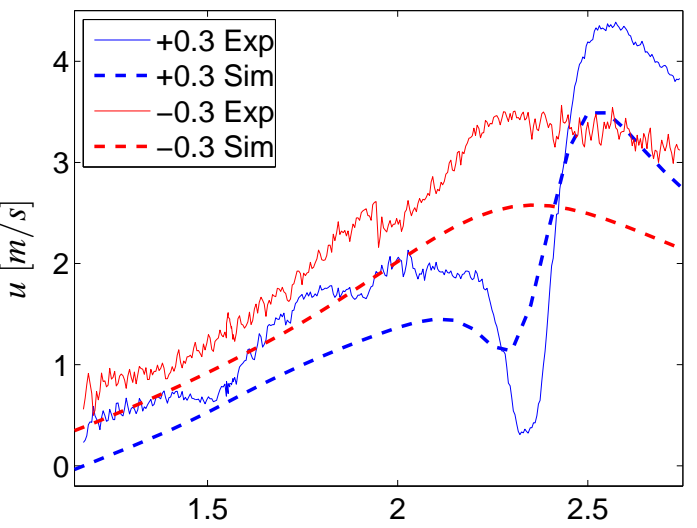

a) Simulation

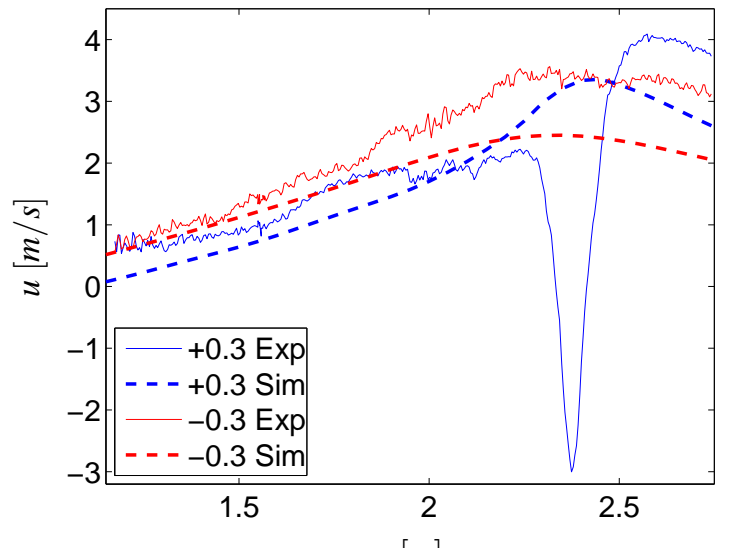

b)

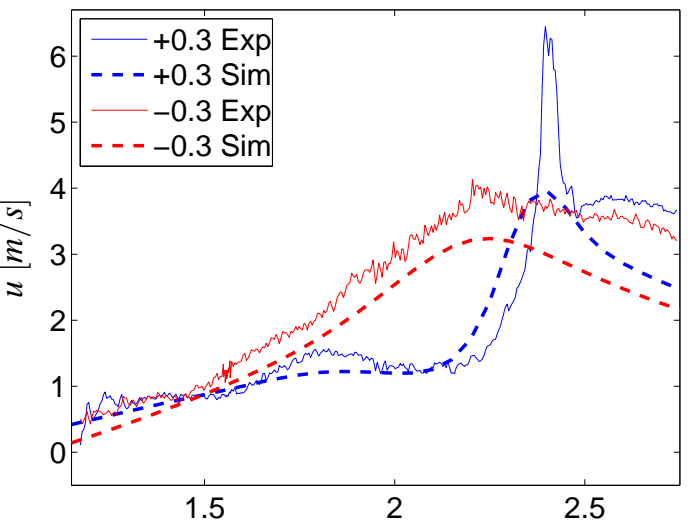

a)

(13. Tangential velocity ( $x$ direction) upstream $(-0.3 \mathrm{~m})$ and downstream $(+\mathbf{0 . 3 m})$ of the rotor, blade position at $40^{\circ}$ and $60^{\circ}$, Exp: Experiment, Sim: Simulation

The tangential velocity component is displayed in figures 12,13 and 14 . The simulation results are verified by the measurement data. The measurements show an abrupt change downstream of the rotor in the vicinity of the blade tip at $r=2.5 \mathrm{~m}$ which makes a considerable peak value for the tangential velocity. Although the simulation also predicts the same peak value at $r=2.5 \mathrm{~m}$, its magnitude is approximately half of the ones found in measurements.

Figures 15, 16 and 17 show a good agreement between the simulation and experiments for the radial velocity component upstream and downstream of the rotor. Upstream of the rotor, there should be no radial velocity component because of the uniform axial incoming flow. However, the passage of rotor through the measurement plane for 20 and 40 degrees make a small radial velocity component. Contrary to the upstream flow, there is a small radial velocity component downstream of the rotor because of the trailing wake vortices. Although its magnitude is small, it makes the trailing wake to expand. Moreover, there is fluctuation in the radial velocity component by experiments. However, the oscillations domain are very small. 


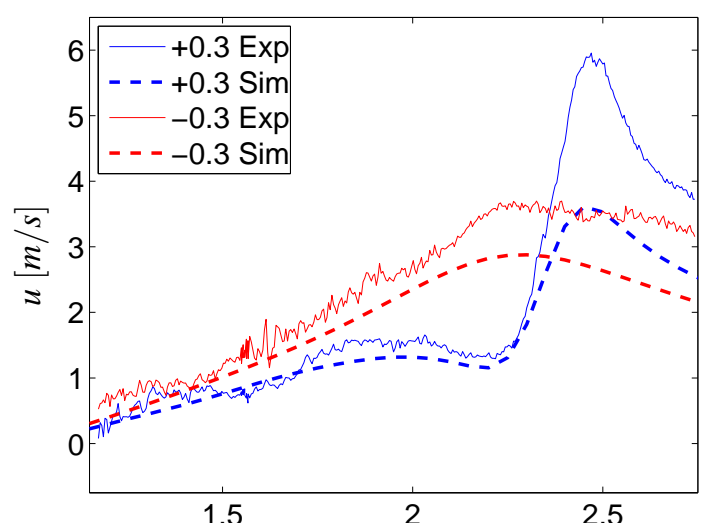

a)

Figure 14. Tangential velocity ( $x$ direction) upstream $(-\mathbf{0 . 3 m})$ and downstream $(+0.3 \mathrm{~m})$ of the rotor, blade position at $80^{\circ}$ and $100^{\circ}$, Exp: Experiment, Sim: Simulation

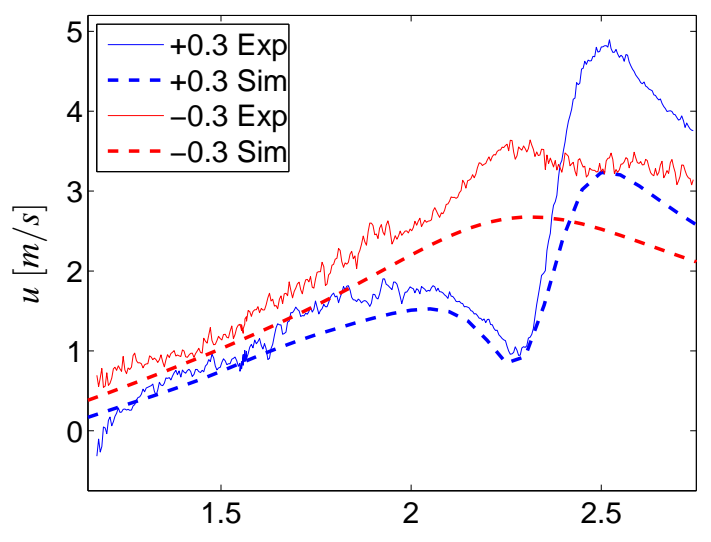

$r[m]$ 


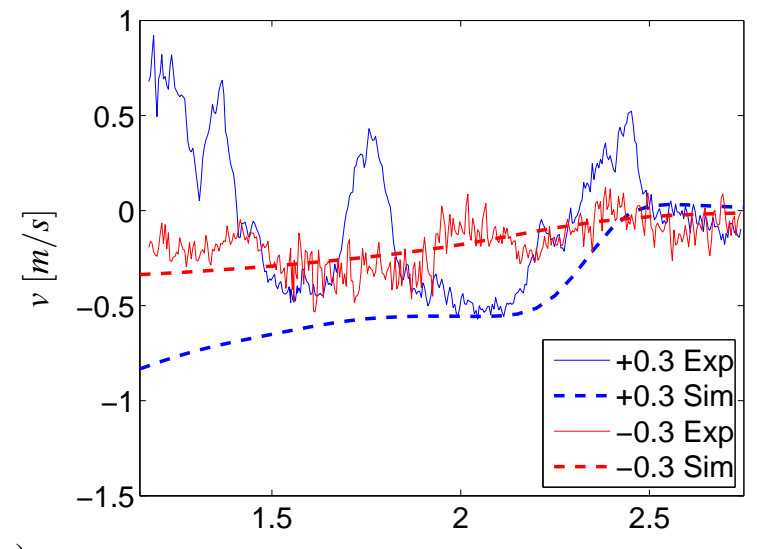

a)

Figure 17. Radial velocity (y direction) upstream (-0.3m) and downstream (+0.3m) of the rotor, blade position at $80^{\circ}$ and $100^{\circ}$, Exp: Experiment, Sim: Simulation

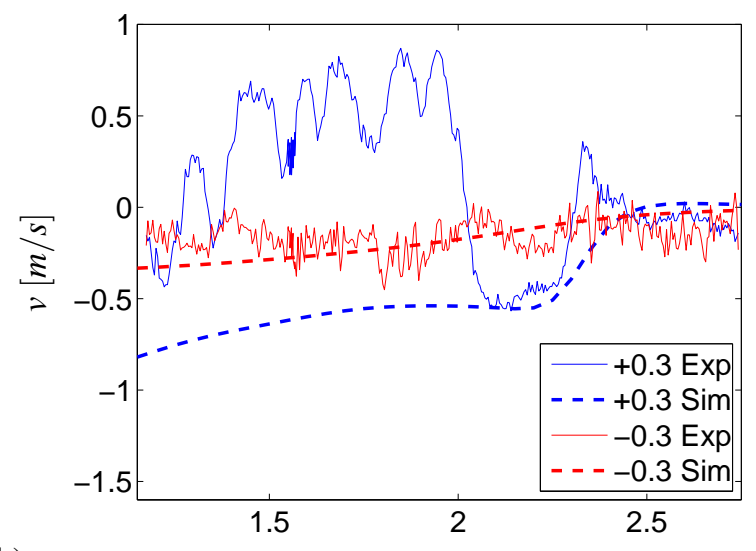

b)

\section{Conclusions}

In this paper the flow field around the MEXICO turbine is studied by VLFW method under the operating conditions i.e., $10.01 \mathrm{~m} / \mathrm{s}$ and $44.45 \mathrm{rad} / \mathrm{s}$ for the incoming wind velocity and the rotational velocity, respectively. The results have been compared with the experimental data where the upstream flow is steady and uniform. However, it should be noted that the upstream velocity deficit in the axial direction in the experiments seems to be too large which may be due to the use of an open wind tunnel type. There is a good agreement between the simulation and the experiments regarding the tip vortex position which varies with respect to the azimuthal angle. But the simulation shows a smaller wake expansion compared with the experiments. Moreover, because of the steady incoming flow perpendicular to the rotor plane, both simulation and measurements confirms that the radial velocity component upstream the rotor is equal to zero.

\section{Acknowledgments}

The data used have been supplied by the consortium which carried out the EU FP5 project Mexico: 'Model rotor EXperiments In COntrolled conditions'.

The Swedish Wind Power Technology Centre (SWPTC) is a research centre for design of wind turbines. The purpose of the Centre is to support Swedish industry with knowledge of design techniques as well as maintenance in the field of wind power. The research in the Centre is carried out in six theme groups that represent design and operation of wind turbines; Power and Control Systems, Turbine and Wind loads, Mechanical Power Transmission and System Optimisation, Structure and Foundation, Maintenance and Reliability as well as Cold Climate. This project is part of Theme group 2.

SWPTCs work is funded by the Swedish Energy Agency, by three academic and thirteen industrial partners. The Region Västra Götaland also contributes to the Centre through several collaboration projects.

\section{References}

${ }^{1}$ Hansen, M. O., Aerodynamics Of Wind Turbines, 2nd edition, EarthScan, 2008.

${ }^{2}$ van Garrel, A., Development Of A Wind Turbine Aerodynamics Simulation Module, ECN report, ECN-C-03-079, August 2003.

${ }^{3}$ Vermeer, L., Sørensen, J., and Crespo, A., "Wind Turbine Wake Aerodynamics," Progress in Aerospace Sciences, Vol. 39, 2003 , pp. 467-510.

${ }^{4}$ Manwell, J., McGowan, J., and Rogers, A., Wind Energy Explained: Theory and Design and Application, John Wiley \& Sons, 2002.

${ }^{5}$ Gupta, S., Development Of A Time-Accurate Viscous Lagrangian Vortex Wake Model For Wind Turbine Applications, University of Maryland, Department of Aerospace Engineering, 2006.

${ }^{6}$ Pesmajoglou, S. and Graham, J., "Prediction Of Aerodynamic Forces On Horizontal Axis Wind Turbines In Free Yaw And Turbulence," Journal of Wind Engineering and Industrial Aerodynamics, Vol. 86, 2000, pp. 1-14.

${ }^{7}$ Voutsinas, S., "Vortex Methods In Aeronautics: How To Make Things Work," International Journal of Computational Fluid Dynamics, Vol. 20, 2006, pp. 3-18.

${ }^{8}$ Garcia, N. R., Sørensen, J., and Shen, W. Z., "Simulations of the Yawed MEXICO Rotor Using a Viscous-Inviscid Panel Method," Journal of Physics: Conference Series, Vol. 524, 2014. 
${ }^{9}$ Garcia, N. R., Sørensen, J., and Shen, W. Z., "Development of a Three-Dimensional Viscous-Inviscid coupling Method for Wind Turbine Computations," Proceedings of the 2013 International Conference on aerodynamics of Offshore Wind Energy Systems and wakes (ICOWES2013), 2013, pp. 569-81.

${ }^{10}$ Chattot, J., "Helicoidal Vortex Model For Wind Turbine Aeroelastic Simulation," Computers and Structures, Vol. 85, 2007, pp. $1072-1079$. 424

${ }^{11}$ Chattot, J., "Optimization Of Wind Turbines Using Helicoidal Vortex Model,” Journal of Solar Energy Engineering, Vol. 125, 2003, pp. 418-

${ }^{12}$ Schepers, J. and Boorsma, K., Description of experimental setup MEXICO measurements, Energy research Centre of the Netherlands, ECN-X-09-0XX, 2006.

${ }^{13}$ Katz, J. and Plotkin, A., Low-Speed Aerodynamics, Cambridge University Press, 2nd ed., 2001.

${ }^{14}$ Abedi, H., Development of Vortex Filament Method for Aerodynamic Loads on Rotor Blades, Licentiate thesis, Department of Applied Mechanics, Chalmers University of Technology, Göteborg, 2013.

${ }^{15}$ Chasapogiannis, P. and Voutsinas, S., "Aerodynamic Simulations of the flow around a Horizontal Axis Wind Turbine using the GAST software, (in Greek)," Mis: 379421, ref.: 68/ 1110, National Technical University of Athens, Department of Mechanical Engineering, Fluid Section, 2014. 